\title{
Examining water in model membranes by near infrared spectroscopy and multivariate analysis
}

\author{
Jorge J. Wenz \\ Instituto de Investigaciones Bioquímicas de Bahía Blanca, Universidad Nacional del Sur (UNS)-CONICET, Bahía Blanca, Argentina \\ Departamento de Biología, Bioquímica y Farmacia, UNS, Bahía Blanca, Argentina.
}

\section{A R T I C L E I N F O}

\section{Keywords:}

Membranes

Water

Near infrared spectroscopy

Principal Component Analysis

\begin{abstract}
A B S T R A C T
By exploiting the sensitivity of the NIR spectrum, particularly the first overtone of water, to the number and strength of hydrogen bonds, the hydrogen bond network and water polymerization in membranes of DMPA (1,2dimyristoyl-sn-glycero-3-phosphate) and DMPC (1,2-dimyristoyl-sn-glycero-3-phosphocholine) was investigated as a function of the temperature and the presence of this two phospholipids having the same tail but different polar head. Principal components analysis performed on the spectra was used to disclose subtle spectral changes that mirror the alteration of the vibrational energy of the water $\mathrm{O}-\mathrm{H}$ bonds, as a measure of the $\mathrm{H}$-bond network. Temperature showed a dominating effect on the H-bond network. Increasing temperatures diminished the number of strongly $\mathrm{H}$-bonded water molecules and increased the number of weakly $\mathrm{H}$-bonded waters. This main effect of temperature was missing after the subtraction of the pure water spectra from the lipid-containing ones. An intriguing secondary effect of temperature was also revealed. Phospholipids exhibited an effect qualitatively similar to that of the temperature. DMPA, and particularly DMPC, disrupted the H-bond network in the neighboring lipid-water interface, reducing water polymerization and strengthening the water $\mathrm{O}-\mathrm{H}$ bonds. The type of the polar head affects the H-bonds more than duplicate the concentration of the lipid. A connection between head group structure and the effect on the H-bonds network, and the existence of two populations of water molecules are discussed.
\end{abstract}

\section{Introduction}

Water in membranes has been an important subject in the study of the phase behavior of phospholipids since the early reports [1-3], and it is considered a paradigm for membrane hydration.

A variety of techniques were employed to study water in membranes. Near infrared spectroscopy (NIR) is a nondestructive, accurate analytical method useful to measure parameters of various systems through their single scans in the 750 and $2500 \mathrm{~nm}$ range $\left(13000-4000 \mathrm{~cm}^{-1}\right)$. It reflects overtones or combinations of fundamental molecular vibrational modes of the $\mathrm{O}-\mathrm{H}$ bond of water and thus informs about $\mathrm{H}$-bonds network. By analyzing the first overtone of water in the 1300-1600 nm NIR region, derived from the main tension band of the $\mathrm{O}-\mathrm{H}$ bond in the middle infrared $(2700-3200 \mathrm{~nm})$, it is possible to investigate the H-bonds network and water polymerization in aqueous systems $[4,5]$. Other regions of the NIR spectra have also been employed for studying water status, as the $1100-1300 \mathrm{~nm}[5,6]$ and $1800-2100 \mathrm{~nm}$ intervals [7]. The $1684-1800 \mathrm{~nm}$ region was recently exploited for studying the chain-melting phase transitions of lipid bilayers $[8,9]$. The NIR water spectrum changes according to the number and strength of hydrogen bonds and the degree of water aggregation [6]. Nearly a century ago [10], these factors were reported as being sensitive to temperature; later, they were also found as being sensitive to solutes that interact with water [11].

Instead of the direct measurement of the component of interest (salt, protein, lipid, etc.) in an aqueous system, the approach used in this work is focused on measuring the changes that the component cause to the water NIR spectra [12]. Even though water absorbs energy in the whole spectra, the NIR region is particularly interesting as the radiation is not totally reflected (as in the visible window) nor totally absorbed (as in the mid infrared) by the water. It is then possible to register the radiation sent it back from the perturbed water. In aqueous systems having abundant $\mathrm{H}$-bonds this approach can be used as an indicator of the effect of a solute. Several components in aqueous systems have been

\footnotetext{
Abbreviations: NIR, near infrared spectroscopy; MIR, mid infrared spectroscopy; MLV, multilamellar vesicles; PCA, Principal Component Analysis; PC, principal component; $\mathrm{n}_{\mathrm{w}}$, number

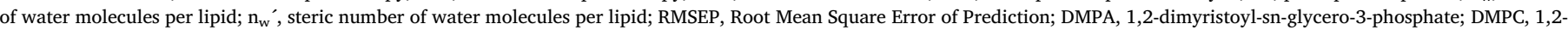
dimyristoyl-sn-glycero-3-phosphocholine

* Camino "La Carrindanga" Km 7, B8000FWB Bahía Blanca, Argentina.

E-mail address: jwenz@criba.edu.ar.
} 
investigated with this methodology: metals [13], sugars [6,7,14], honey [15], milk [16], DNA [17], plants [18], inorganic salts [4,11], acids and hydroxides [11], cells and tissues [12,19].

A required step in this line of attack is the use of multivariate analysis to disentangle the subtle and hidden spectral perturbations. Broad and overlapped bands are usual and preprocessing procedures as smoothing, derivatives, mean centering, autoscaling, etc., are commonly used for the discrimination and interpretation of the spectra. Then, one or more multivariate techniques, as Principal Component Analysis (PCA), Partial Least Squares (PLS), Multiple Linear Regression (MLR), Soft Independent Modeling of Class Analogy (SIMCA), etc., are usually applied. Last but not least, the outcomes requires a meticulous examination for a correct interpretation [20,21]. See Supplementary material for a more detailed explanation of the multivariate method employed in this work, PCA.

As far as the author is aware, no work using NIR spectroscopy for the study of water in membranes has been reported. NIR spectroscopy and multivariate analysis of the spectra were combined here to investigate the effect of temperature and phospholipids on the hydrogen bond network and water aggregation in membranes. For that, multilamellar vesicles (MLV) of phospholipids having an identical fourteen carbon acyl chains but polar heads differing in the presence of an hydroxyl (phosphatidic acid) or a choline group (phosphocholine), were prepared at two concentrations and scanned at varying temperatures.

\section{Experimental procedure}

\subsection{Materials}

The DMPA (1,2-dimyristoyl-sn-glycero-3-phosphate) and DMPC (1,2-dimyristoyl-sn-glycero-3-phosphocholine) were purchased from Avanti Polar Lipids (Alabaster, AL), having a purity $>99 \%$. Stock solutions were prepared in chloroform/methanol $(2: 1 \mathrm{v} / \mathrm{v})$ at a final concentration of $1 \mathrm{mg} / \mathrm{ml}$. All solutions were stored at $-20^{\circ} \mathrm{C}$ before use.

\subsection{Liposome preparation}

Multilamellar vesicles (MLV) were prepared by mixing the appropriate amount of stock solution of each component in round-bottomed tubes and dried under nitrogen flow at room temperature while rotating the tube. The samples were then dried under nitrogen flux for $1 \mathrm{~h}$ to eliminate the remaining solvent. Milli-Q water was added to the thin lipid film and heated up to $55-57^{\circ} \mathrm{C}$, i.e., at a temperature higher than the highest transition temperature $\left(\mathrm{T}_{\mathrm{m}}\right)$ of the lipids. At this stage, the total lipid concentration of the suspension was around $1 \mathrm{mg} / \mathrm{ml}$. The samples were then vigorously mixed in a vortex in two steps of $1 \mathrm{~min}$ each, also at $55-57^{\circ} \mathrm{C}$, and incubated for $30 \mathrm{~min}$ in a low power bath sonicator in order to obtain a more uniform size distribution of the resulting MLVs. Lipid suspensions were then diluted with Milli-Q water to a final concentration of 250 and $500 \mu \mathrm{M}$.

\subsection{Spectroscopic measurements}

Near infrared spectra were obtained in a Brimrose Solid-state Luminar 5030 AOTF-NIR (Acousto-Optic Tunable Filter-Near Infrared) Analyzer (Brimrose, Sparks, MD), equipped with a probe attachment and a spectra analysis software (Snap32). For each measurement the probe with its reflectance attachment was immersed directly into the round-bottomed tubes containing $1.5 \mathrm{ml}$ of the lipid suspension. Absorbance $\left(\log \mathrm{T}^{-1}\right)$ was registered every $1 \mathrm{~nm}$ in the interval $1100-2300 \mathrm{~nm}$, with a layer of thickness of the suspension of $1 \mathrm{~mm}$, corresponding to the gap of the immersion probe. Samples were scanned every $5{ }^{\circ} \mathrm{C}$ in the range $13-58^{\circ} \mathrm{C}$ in a temperature-controlled circulating water bath (Cole Parmer Polystat) with a heating rate of $0.4^{\circ} \mathrm{C} / \mathrm{min}$. Special care was taken for the temperature control. Due to the relatively high ratio between the mass of the probe $(\mathrm{a} 20 \mathrm{~cm}$ length $\times 1,2 \mathrm{~cm}$ diameter stainless steel cylinder) and that of the samples to be measured $(1.5 \mathrm{ml} \approx 1.5 \mathrm{~g}$ ), a significant thermal transference from (or to) the sample may occur, depending on the direction of the heat flux. To minimize possible temperature fluctuation, the probe was maintained submerged into the bath. Before sample measurement, the probe was rinsed with Milli-Q water (also at the working temperature) and further dried with tissue paper to prevent dilution effects. Each sample, with the probe inside the tube, was kept in the water bath during the spectra acquisition.

A total of sixty spectra involving samples/conditions were registered: two control samples (Milli-Q water), two different liposomes (DMPA and DMPC) at two concentrations (250 and $500 \mu \mathrm{M}$ ), and 10 temperatures ( 13 to 58 , every $5{ }^{\circ} \mathrm{C}$ ). Each of the sixty spectra was the average of 5 scanning of the corresponding sample/condition. They were averaged in view that the variation coefficient $(\mathrm{SD} * 100 /$ Mean) of the absorbance at each wavelength was $<0.2 \%$.

\subsection{Multivariate analysis of NIR spectra}

\subsubsection{Principal Component Analysis}

Depending on the objective, PCA was performed on different set of samples and on different spectral windows using The Unscrambler [22]. Prior to PCA, a mean centering was performed on the raw spectra. PCA was firstly applied to the raw spectra of the matrix of 60 samples (including type of phospholipid, concentration and temperature) and 1201 variables (wavelengths). PCA was also performed on separated set of samples composed of water, DMPA and DMPC liposomes. PCA was also performed on spectra after the subtraction of the pure water spectrum at the corresponding temperature. More details about the PCA procedure can be found in the Supplementary material ("Principal Component Analysis").

\section{Results}

\subsection{Raw NIR spectra of water}

Fig. 1 shows the sixty NIR spectra corresponding to two control samples (Milli-Q water), DMPA and DMPC liposomes at two concentrations $(250$ and $500 \mu \mathrm{M})$ measured every $5{ }^{\circ} \mathrm{C}$ from 13 to $58^{\circ} \mathrm{C}$ (ten temperatures). Spectra were very similar and it was almost unfeasible to distinguish visually between samples according to the type of phospholipid (DMPA or DMPC) or concentration. The effect of temperature, illustrated by the arrows in Fig. 1, is the only spectral trend that can be ascertained by an examination at specific regions of the spectra. The main of the first overtone of water reflecting $\mathrm{O}-\mathrm{H}$ stretching is centered around 1450-1455 nm, in agreement with other reports [23-25]. An isosbestic region is observed at 1425-1430 nm, not far from the 1440-1442 nm [26] and $1446 \mathrm{~nm}$ [24] reported for pure water. This band, as well as others of the NIR spectra, may shift depending on the interaction with the solute. In the presence of inorganic salt, for example, [4] observed the isosbestic point quite far from these values, at $1497 \mathrm{~nm}\left(6680 \mathrm{~cm}^{-1}\right)$. Increasing temperatures increase the absorbance on the left shoulder of the band and it decreases toward the right shoulder (Fig. 1), entailing a shift to shorter wavelengths (a "blue shift"), i.e. higher energy. This means that temperature weakens the $\mathrm{H}$ bonds and this strengthens the $\mathrm{O}-\mathrm{H}$ bonds; then, they vibrate and absorbs radiation at higher energy [24,27]. In other words, when temperature increases, the fraction of weakly bonded water increases, while that of strongly bonded water decreases.

\subsection{Temperature and phospholipid effects on water are reflected in the NIR spectra}

From the abundant PCA output, only those providing useful information for the present purposes are discussed in the next sections. 


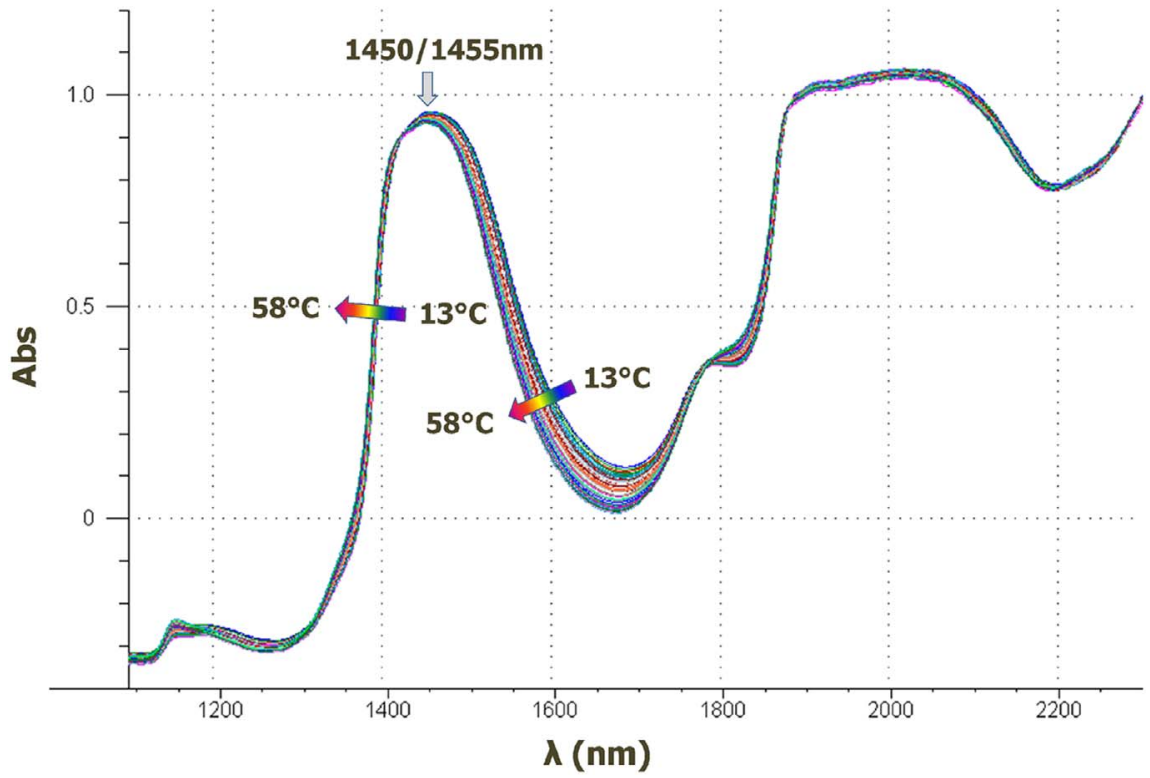

Fig. 1. The sixty NIR spectra analyzed in this work: two control samples (Milli-Q water), two phospholipids samples (DMPC and DMPA) at two concentrations ( 250 and $500 \mu \mathrm{M})$, all measured at ten temperatures (every $5{ }^{\circ} \mathrm{C}$ in the $13-58^{\circ} \mathrm{C}$ interval). Each spectrum emerges from the average of five readings of the same sample/condition. The variation coefficient of the absorbance at each wavelength was $<0.2 \%$ between the five scanning.
Measurements corresponding to water at $43^{\circ} \mathrm{C}$ were found as an outlier by the PCA and it was thus removed from the analysis.

By means of PCA it was possible to reduce the dimensionality of the original data (1201 variables) to three orthogonal components (PCs), which were able to explain $99.8 \%$ of the total variability (PC1, 96.8\%; PC2, 2.9\%; PC3, 0.1\%). A percentage quoted close to a PC specifies the percentage of variability explained by the PC.

Samples in a score plot are placed according to their similarities (or differences) and the distance between them is proportional to differences in the spectrum. No information other than the spectrum is employed during the model construction. Fig. 2A shows the distribution of samples in the PC2/PC1 plane; the connecting lines indicate the sample evolution with temperature, increasing $5{ }^{\circ} \mathrm{C}$ from one point to the next toward the left. PC1 describes the variation due to the temperature along the horizontal axis. The vertical spread between the series signifies that PC2 captures and discriminates the effect of phospholipids. The need of an extra PC to explain the spectral changes implies that phospholipids perturb the H-bond network and the water aggregation in a way different than that caused by temperature. Considering the high percentage of explanation of PC1 $(96.8 \%)$ is evident that temperature is, by far, the dominating perturbing factor. Note that equivalent temperatures in the five series of Fig. 2A (water, DMPA and DMPC at two concentrations) are nearly aligned vertically, denoting similar PC1 scores and thus a similar effect of temperature on each sample. Samples are spread horizontally by effect of the temperature and vertically by the type of phospholipid and concentration. Given the increasing proximity between series toward the left side of Fig. 2A, the effect of phospholipids (particularly DMPA) on H-bond network and water aggregation becomes less significant conforming temperature increases. Relative to the pure water series, the vertical distinction along PC2 informs on the magnitude of the effect of the phospholipid. The effect is more noticeable for the highest concentration of both phospholipids, and for DMPC relative to DMPA.

An intriguing secondary effect of temperature was revealed when plotting PC3 versus PC1 scores (Fig. 2B). Despite the small percentage of variability explained by PC3 $(0.1 \%)$ compared to that of the temperature $(96.8 \%)$, a clear parabolic shape with a turning back point around $33-38^{\circ} \mathrm{C}$ was detected in all samples. The necessity of an additional principal component (PC3) indicates the existence of a different effect of the temperature, reflected by further spectral changes in the multidimensional space of the 1201 variables different from those captured by PC1. The parabolic pattern is roughly symmetric around the maximum, and then PC3 scores have similar values for highest and the lowest temperatures equidistant from the center. This secondary effect of temperature keeps a tendency from $13^{\circ} \mathrm{C}$ up to $33-38^{\circ} \mathrm{C}$ and then it goes back, showing at $58{ }^{\circ} \mathrm{C}$ values of PC3 similar to those at $13{ }^{\circ} \mathrm{C}$. Despite of the overlapping of series in Fig. $2 \mathrm{~B}$, indicative of a comparable secondary effect of temperature on all samples, the turning point temperature shows a slight variation depending on the sample (Fig. 3B, shaded areas). This behavior is quite different from the monotonous trend of the main effect of temperature (Fig. 2A). The second effect of temperature will be addressed later is Section 4 Discussion.

\subsection{Analysis of spectral regions}

\subsection{1. $1100-2300 \mathrm{~nm}$}

PCA outcome includes the loadings, a parameter that informs about the weight and inter-correlation between variables. By contrasting the sign of loadings with the sign of scores relationships between variables and samples can be found [28]. If a wavelength has a positive loading on a PC it means that all samples with positive scores on that PC have higher than average values of absorbance at that wavelength; on the contrary, samples with negative scores have lower than average values at that wavelength. If a wavelength has a negative loading, it means the opposite. Samples with positive scores have lower than average values at that wavelength, while samples with negative scores have higher than average values at that wavelength. If the number of samples plus variables is small (which is not the present case) this can be readily ascertained by placing scores and loading in the same plot. The proximity between a sample (scores) and a wavelength (loadings) means that such sample has an absorbance higher than the average at that wavelength. This approach was utilized to ascertain the wavelengths that better capture the effect of temperature and phospholipids on the $\mathrm{H}$ bonds network and the water aggregation. The rationale is also valid in quantitative terms, as the higher the positive score of a sample, the larger its values for wavelengths with positive loadings, and vice-versa $[20,21]$. The search for absorption bands that better reflect the changes in the water status was focused in those wavelengths having high or low loadings (i.e. far from zero) and located in regions equivalent to that of the samples in the PC1/PC2 space (Fig. 2A). It is worth noticing that it is not the absorption peak shown in Fig. 1 what is determined by this procedure, but the wavelengths at which the absorption varies most, and so better discriminate between samples. Peaks in the loading plots cannot be directly related to absorbance peaks, as can be seen by comparing peaks in Fig. 1 and Fig. 2C. 

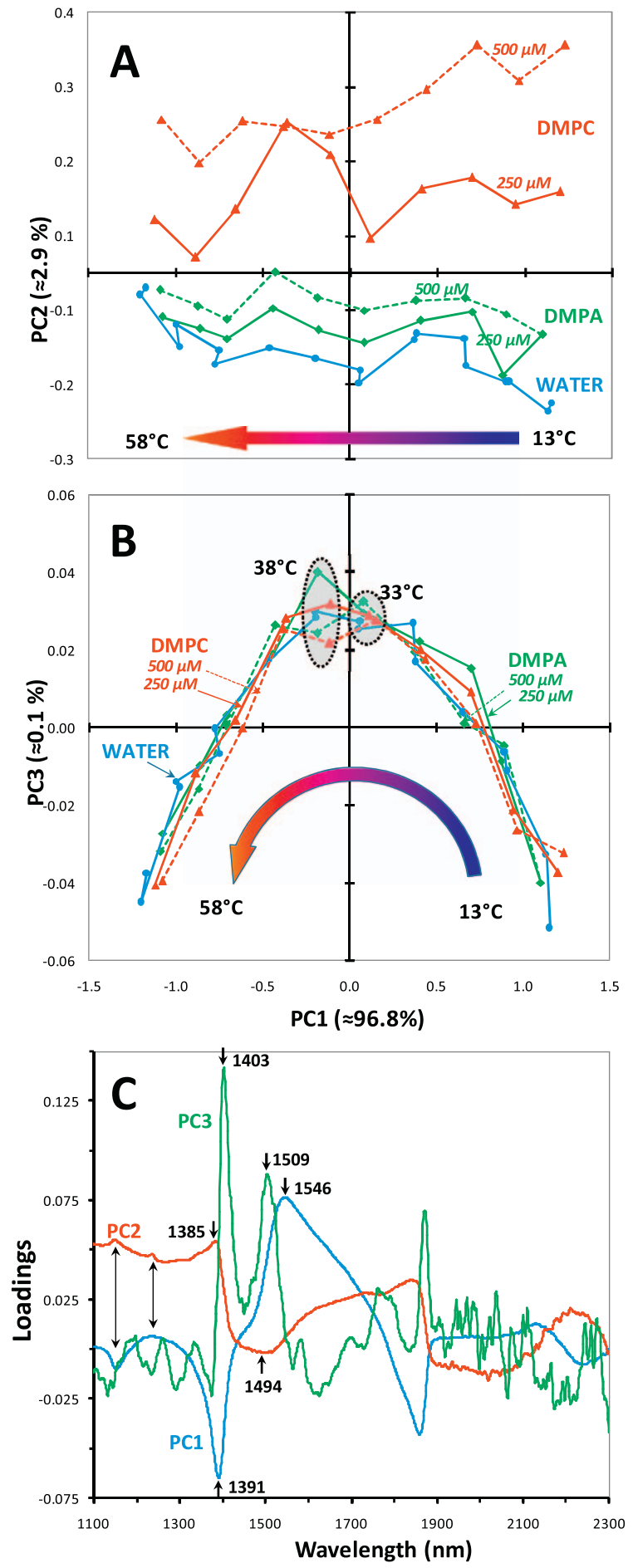

Fig. 2. Scores plot from PCA performed on the raw spectra of all samples in the entire range $(1100-2300 \mathrm{~nm})$. Panel A: PC2 vs. PC1; Panel B: PC3 vs. PC1. The evolution of samples with temperature is shown by connecting lines. As indicated by the arrow, temperature increases from right to left (with a $5{ }^{\circ} \mathrm{C}$ step between points). Percentages of the total variance explained are shown in brackets nearby each PC. The pure water sample at $43{ }^{\circ} \mathrm{C}$ was removed from the analysis as it was found as an outlier. Panel C: loadings from the PCA.

The loadings for the first three PCs are shown in Fig. 2C. Arrows point out the wavelengths of maximum absorbance variation, involving both positive and negative loadings. For example, PC1 loadings (blue line) are negative at wavelengths around $1391 \mathrm{~nm}$; concurrently, PC1 scores are also negative for samples at the highest temperatures, i.e. those toward the left side of Fig. 2A and Fig. 2B. This implies that
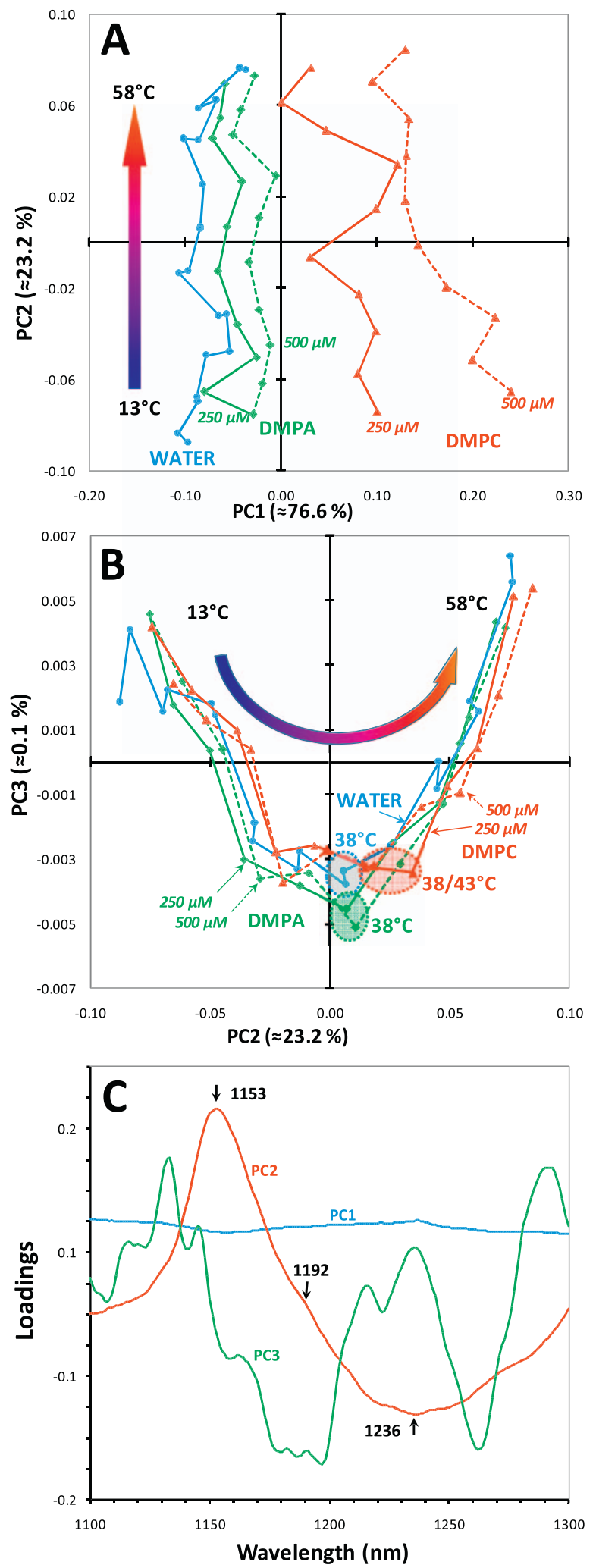

Fig. 3. Scores plot from PCA performed on the raw spectra of all samples in a limited spectral region (1100-1300 nm). A: PC2 vs. PC1; B: PC3 vs. PC2. The evolution of a sample with temperature is illustrated with connecting lines. Temperature increases in the direction of the arrows every $5{ }^{\circ} \mathrm{C}$ between points. Percentages of the total variance explained by each PC are shown in brackets. The pure water sample at $43{ }^{\circ} \mathrm{C}$ was removed from the analysis as it was found as an outlier. Panel C: loadings from the PCA. 
increasing temperatures increase the absorbance mainly at $1391 \mathrm{~nm}$, i.e. on the left of the $1450 / 1455 \mathrm{~nm}$ band. On the other hand, PC1 loadings around $1546 \mathrm{~nm}$ and PC1 scores at the lowest temperatures (toward the right side on Fig. 2A and Fig. 2B) are both positive, indicating that increasing temperature decrease the absorbance at $1546 \mathrm{~nm}$. This rationale of contrasting loadings and scores can be extended for the rest of PCs, samples and variables. The effect of phospholipids is captured by PC2. The presence of DMPA and particularly DMPC is correlated with high PC2 loadings at $1385 \mathrm{~nm}$ (Fig. 2C, red line) and with high PC2 scores (Fig. 2A), indicating that phospholipids increase in the absorbance mostly at $1385 \mathrm{~nm}$, i.e. on the left shoulder of the water first overtone band. As mentioned, PC3 captures a secondary effect of temperature with a turning point at $33-38^{\circ} \mathrm{C}$. Contrasting the positive PC3 scores (Fig. 2B) around the turning point and the PC3 loadings (Fig. 2C, green line), this effect of temperature involves an increase in the absorbance mainly at 1403 and $1509 \mathrm{~nm}$. The preceding results indicate that both the temperature and the presence of DMPA and specially DMPC, disrupt the water H-bond network and thus strengthen the $\mathrm{O}-\mathrm{H}$ bonds, leading to a lower degree of water polymerization.

\subsection{2. $1100-1300 \mathrm{~nm}$}

PCA was next performed in a limited spectral range $(1100-1300 \mathrm{~nm})$. The absorbance at these shorter wavelengths have been reported to inform also on the relative proportions of water species having different numbers of $\mathrm{H}$-bonds [5]. Interestingly, PC1 (76.6\%) discriminates mostly the effect of phospholipids (Fig. 3A), instead of the effect of temperature as it does when considering the full NIR spectra. The positive PC1 scores for DMPC samples (Fig. 3A) concurrently with their positive PC1 loadings (Fig. 3C, blue line) indicate that the presence of DMPC increases the absorbance in the entire 1100-1300 nm interval, being the effect more notorious for the highest concentration $(500 \mu \mathrm{M})$. Although to a lesser extent, DMPA causes similar changes (Fig. 3A). The temperature is the second perturbing factor in this spectral interval, described by PC2 (23.2\%). The positive PC2 scores for the highest temperatures concurrently with the PC2 loading profile (Fig. 3C, red series) indicate increasing temperature increases the absorbance mainly around $1153 \mathrm{~nm}$ and it decreases around $1236 \mathrm{~nm}$, whereas it keeps constant at the isosbestic region around $1192 \mathrm{~nm}$. These three values of PC2 loadings match well with those associated with the absorption of three forms of water depending on the number of H-bonds, early reported by Bujis and Choppin (1963): water molecules with neither $\mathrm{OH}$ group bonded absorbing at $1160 \mathrm{~nm}$, waters with one $\mathrm{OH}$ group $\mathrm{H}$-bonded absorbing at $1200 \mathrm{~nm}$, and water with two OHs H-bonded absorbing at $1250 \mathrm{~nm}$. Accordingly, conforming temperature increases, the number of molecules bonded with two H-bonds water decreases at a rate equivalent to that of the increase of the number of molecules with no H-bond. The number of molecules with one H-bond remains almost constant since molecules with one and molecules with two $\mathrm{H}$-bonds lose one $\mathrm{H}$-bond at a similar rate. It is worth noticing the presence of two small peaks close to $1153 \mathrm{~nm}$ and $1236 \mathrm{~nm}$ in loadings plot of PCA performed with the entire NIR spectra, denoted with double arrows in Fig. 2C. This concurs with previous finding indicating that both temperature (PC1, blue line) and phospholipids (PC2, red line) have an effect on the water H-bonded network and then modifies the proportions of the different water aggregates.

The score plot of PC3 $(0.1 \%)$ versus PC2 $(23.2 \%)$ also exhibits a parabolic shape in the $1100-1300 \mathrm{~nm}$ interval (Fig. 3B), recalling the previous findings from the full spectra (see Section 3.2. Temperature and phospholipid effects on water are reflected in the NIR spectra Interestingly, the two PCs involved in the parabolic profile describe temperature effects, in both circumstances: PC3/PC1 in the $1100-2300 \mathrm{~nm}$ interval and PC3/PC2 in the $1100-1300 \mathrm{~nm}$ interval. As stated, the turning point varies slightly with the type of sample, having DMPC the highest turning back temperature (Fig. 3B, shaded areas).
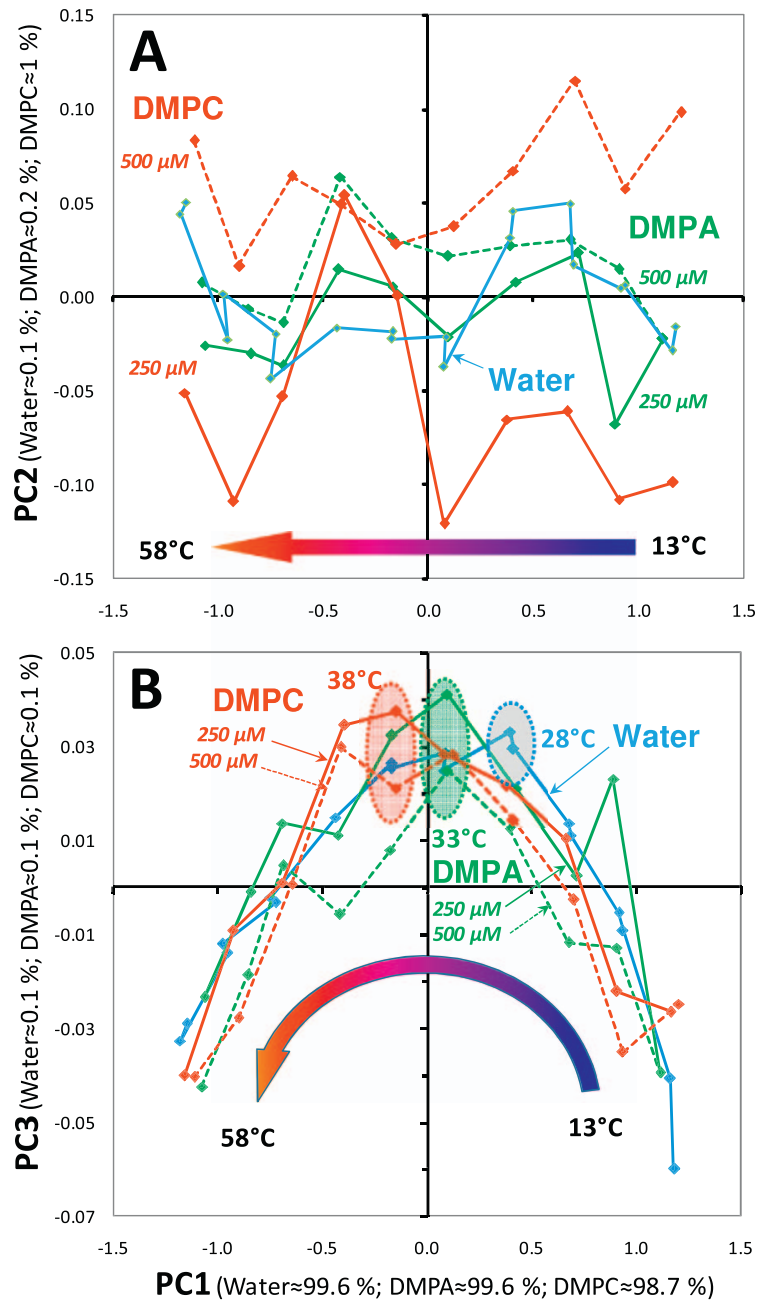

Fig. 4. Scores plot from PCA performed separately on the raw spectra of each group of samples: pure water, DMPA and DMPC. Panel A: PC2 vs. PC1; Panel B: PC3 vs. PC1. Lines connecting points denote the evolution of a sample with temperature. Temperature increases as shown by the arrows, with a $5{ }^{\circ} \mathrm{C}$ step between points. Percentages of the total variance explained by each PC are shown in the axes. The shaded areas show the turning point of the effect of temperature for each group of samples. The pure water sample at $43^{\circ} \mathrm{C}$ was removed from the analysis as it was found as antlier.

\subsection{PCA performed separately on pure water, DMPA and DMPC samples}

PCA was next performed individually on samples of pure water, DMPA and DMPC, at 250 and $500 \mu \mathrm{M}$, and in the entire NIR interval $(1100-2300 \mathrm{~nm})$. As in the entire sample set, PC1 describes the main effect of temperature and explains most of the spectral variability (water: 99.6\%, DMPA: 99.6\%; DMPC: 98.7\%) within each group (Fig. 4A). As expected, the explained variance is higher than that for PCA performed on all samples (PC1, 96.8\%), as the variability due to different phospholipids is missing. PC2 discriminates between concentrations of each lipid and thus its contribution to the explained variance (DMPA: $0.2 \%$; DMPC: $1.0 \%$ ) is smaller than that of the entire set $(2.9 \%)$. Given that subsequent PCs are always selected following a decreasing order of explaining capability (PC1, PC2, and so on), these differences in the percentage of explanation of PC2 for separated and grouped analysis indicates that the effect of different head group on the H-bonds network and water aggregation is more significant than the effect of double their concentrations $(250$ and $500 \mu \mathrm{M})$ of the same lipid.

The PC3/PC1scores plot for the separated sample set exhibit a parabolic shape with a turning point (Fig. 4B), similar to that for the entire sample set (Fig. 2B). Even though differences between pure 
water, DMPA and DMPC series in Fig. 4B are not considerable (series are nearby), PC3 scores reach the turning point at temperatures that slightly vary with the type of sample: water $28^{\circ} \mathrm{C}$, DMPA $33^{\circ}$ and DMPC $38^{\circ} \mathrm{C}$ (Fig. 4B, shaded areas). These results concur with the previous findings from the analysis of the entire sample set (Section 3.2. Temperature and phospholipid effects on water are reflected in the NIR spectra) and from the analysis of a restricted spectral window (Section 3.3. Analysis of spectral regions), and suggest that the secondary effect of temperature is sensitive to the presence of phospholipids.

\subsection{The main effect of temperature is missing by removing the pure water spectra}

The NIR spectra comprise signals from all water molecules, including those in the lipid-water interface and those in the bulk. Whereas both populations are perturbed by the temperature, only water molecules at the interface are supposed to be perturbed by the neighboring phospholipids. To investigate the changes in each population of molecules, the pure water spectrum was subtracted from each phospholipidcontaining sample at each temperature. Although spectral corrections for changes in the molar fraction of the absorbing water molecules caused by changing solute concentration have been suggested [25], it was omitted given the very low concentration of phospholipids: the water:lipid molar ratio is more than one hundred thousand. Because a slight noise appeared after the subtraction process, a smoothing Savitzky-Golay procedure using a 2nd order polynomial with a 24 points window was applied to the difference spectra. Two doubtful samples derived from the pure water at $43{ }^{\circ} \mathrm{C}$ were removed from the analysis, as previously detected as an outlier.
The difference spectra (Fig. 5) look quite different from the raw ones (Fig. 1), and the variation between DMPA and DMPC samples and between the two assayed concentrations can be perceived by visual inspection. This is not unexpected, as they mirror only the water molecules perturbed by the phospholipids in at the interface. If, on the contrary, water in the DMPA and DMPC samples would be unaffected, the subtraction of the pure water signal should give a straight line [7]. Two regions approaching zero are visible around 1440 and $1880 \mathrm{~nm}$ (Fig. 5A), which match the isosbestic regions in the raw spectra with almost constant absorbance (Fig. 1). The absorbance at $1440 \mathrm{~nm}$ is very close to the $1438 \mathrm{~nm}$ reported by Segtnam and co-workers [24] as being representative of a water species whose concentration is relatively constant as a function of temperature; the authors have admitted, however, the absence of unambiguous proof about the nature of such species.

The pure water spectra represent the signal of the bulk water, i.e. molecules far enough from the lipid-water interface for being perturbed by phospholipids. After the subtraction process, the spectral differences between samples become more significant (Fig. 5) than in the raw spectra (Fig. 1). The signal from the large population of water molecules in the bulk is missing, and only the signal emerging from the water molecules at the lipid-water interface contributes to the difference spectra.

\subsection{1. $1100-2300 \mathrm{~nm}$}

Fig. 6A shows the scores plot from PCA performed on the difference spectra over the spectral range $(1100-2300 \mathrm{~nm})$. PC1 $(87.6 \%)$, discriminates mostly between phospholipids and segregates two groups of samples, corresponding to DMPA (green series) and DMPC (red series);
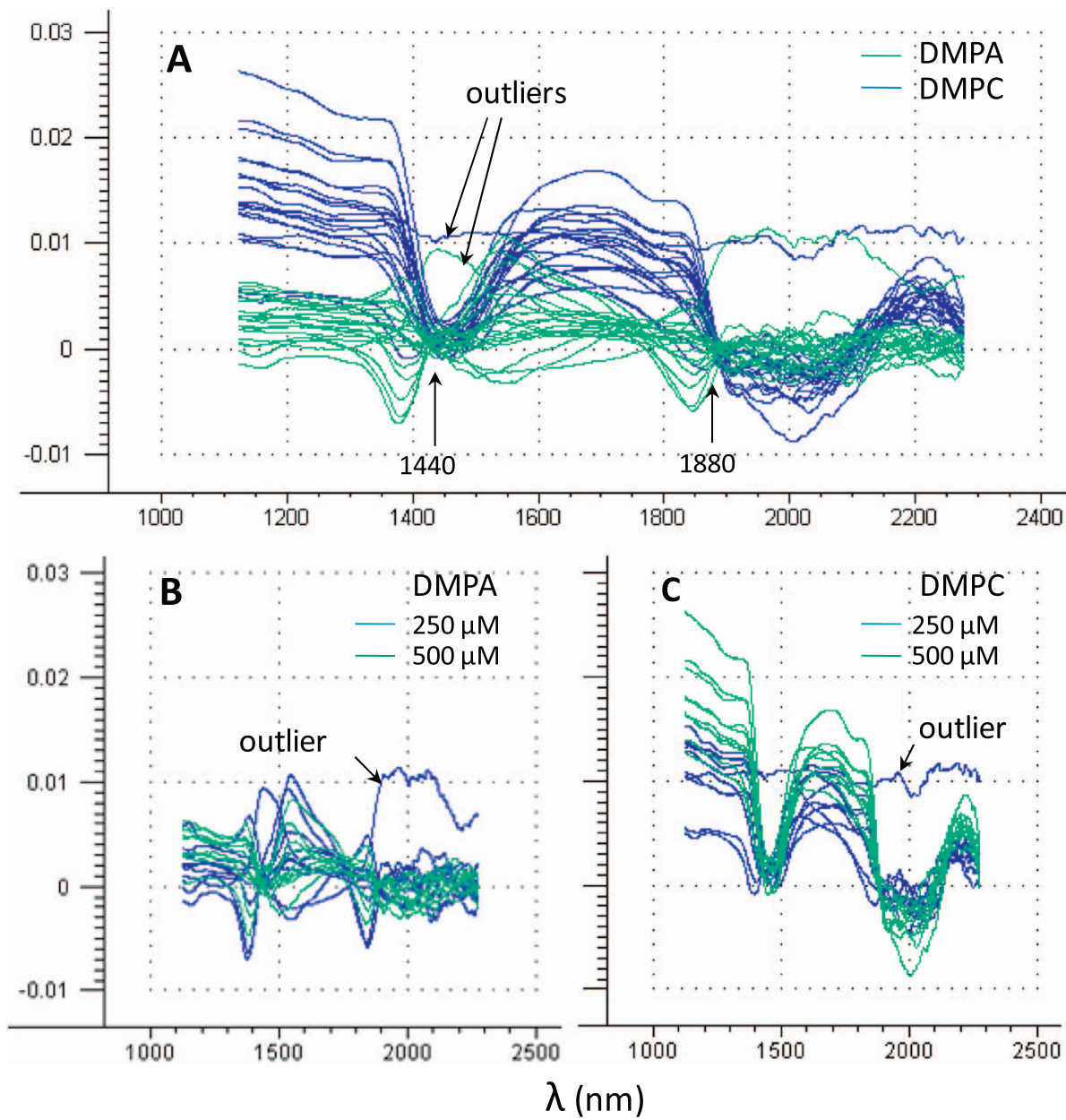

Fig. 5. Difference spectra obtained by subtraction of the pure water spectra from the DMPA and DMPC spectra, at each corresponding temperature. The difference spectra were smoothed throughout the Savitzky-Golay procedure with a 2nd order polynomials and a 24 points window. Panel A: the twenty spectra of DMPA (green) and the twenty spectra of DMPC (blue) samples, including the 250 and $500 \mu \mathrm{M}$ concentration and the ten assayed temperatures $\left(13\right.$ to $58^{\circ} \mathrm{C}$, every $5^{\circ}$ ) for both phospholipids. Panel $\mathrm{B}$ and $\mathrm{C}$ : the twenty spectra of DMPA and DMPC, respectively, at $250 \mu \mathrm{M}$ (blue) and $500 \mu \mathrm{M}$ (green), including the ten assayed temperatures. (For interpretation of the references to color in this figure legend, the reader is referred to the web version of this article.) 

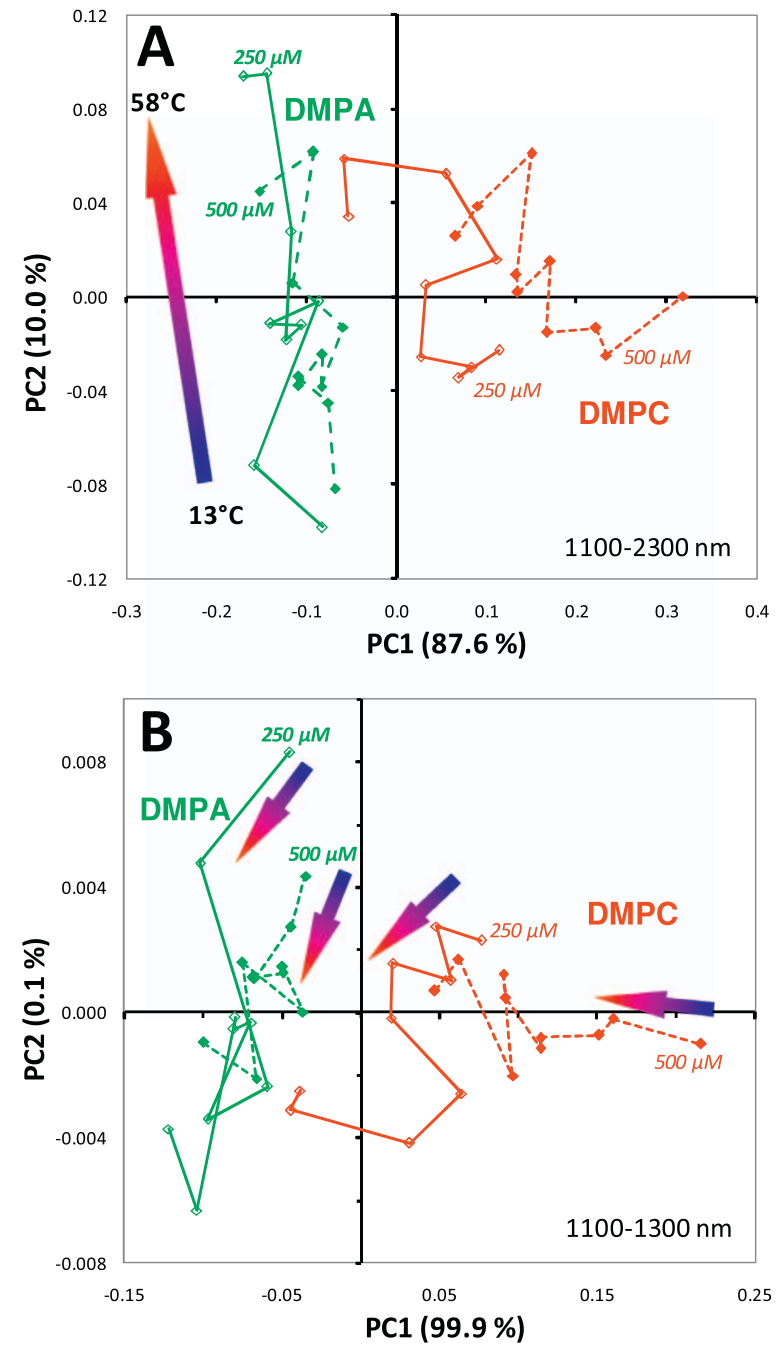

Fig. 6. Scores plot from PCA performed on the difference spectra corresponding to DMPA and DMPC, in the 1100-2300 nm spectral interval (Panel A) and 1100-1300 nm interval (Panel B). Temperature increases from 13 to $58^{\circ}$ as indicated by the arrows along the connecting lines of the series $\left(5^{\circ} \mathrm{C}\right.$ per point). Numbers in brackets on axis label indicate the percentages of the total variance explained by the PC.

the two series within each group correspond to each lipid concentration. Although differences between the two DMPA concentrations (solid and dotted green series) are small, different degrees of perturbation of the water was observed for the two concentration of DMPC (solid and dotted red series). Temperature becomes the succeeding factor of spectral variation, as it is described mostly by PC2 (10.0\%); the evolution of the samples with temperature is denoted by the arrow in Fig. 6A. The increasing proximity between DMPA and DMPC series toward the upper left corner of Fig. 6A suggests that the differences in the H-bond network and the water polymerization at the interface between DMPA and DMPC liposomes become smaller conforming temperature increases, as discussed in Section 3.2. Temperature and phospholipid effects on water are reflected in the NIR spectra No clear trend with varying temperature, phospholipids or concentration was captured by PC3 (1.3\%), implying that the third PC is mainly modeling noise (data not shown). At this point, there is no direct clue to assert if the effect of temperature captured in the difference spectra corresponds to the aforementioned secondary effect of temperature, typically revealed with a parabolic pattern in the score plot. Together with the missing of main effect of temperature, the associated PC1 is also missing. As will be discussed in Section 4. Discussion, two PCs describing temperature effect might be required to capture the typical
Table 1

Principal components capturing the effects of temperature and phospholipids in the raw and in the difference spectra, in the $1100-2300 \mathrm{~nm}$ interval. The difference spectra were obtained by subtracting the pure water spectra from the raw spectra. Numbers in brackets are the percentage of variability explained by the PC.

\begin{tabular}{lll}
\hline & Raw spectra & Difference spectra \\
\cline { 2 - 3 } & Described by & Described by \\
\hline Main effect of temperature & PC1 $(96.8 \%)$ & N/C \\
Effect of phospholipids & PC2 $(2.9 \%)$ & PC1 $(87.6 \%)$ \\
Secondary effect of temperature & PC3 $(0.1 \%)$ & PC2 $(10.0 \%)$ \\
\hline
\end{tabular}

parabolic profile observed in the score plot.

\subsection{2. $1100-1300 \mathrm{~nm}$}

Performing PCA on the difference spectra but in the selected $1100-1300 \mathrm{~nm}$ range, PC1 also captures the effect of phospholipids and discriminates between DMPA and DMPC liposomes (Fig. 6B); PC1 captures same differences between the high and low concentrations of DMPC. The high percentage of explained variability of PC1 (99.9\%) indicates that phospholipids have a substantial effect in this region. PC2 $(0.1 \%)$ mainly captures an effect of the temperature, different that the already mentioned main effect.

The hypothesis of the missing main effect of temperature after the subtraction of the pure water spectra is supported by the inversion in the order of the PCs when comparing PCA performed with the raw and with the difference spectra (Table 1). As aforementioned, the main effect of temperature is described by PC1 $(96.8 \%)$ in the raw spectra and by PC2 (10.0\%) in the difference spectra. On the other hand, the effect of phospholipids is described by PC2 $(2.9 \%)$ in the raw spectra and PC1 $(87.6 \%)$ in the difference spectra, becoming the most important perturbing factor when the main effect of temperature is missing. Hence, the most important PC, responsible for capturing the major source of variation, captures the effect of phospholipids, whereas PC2 captures another temperature effect. Note that when the main effect of temperature is missing, the remaining effect of temperature does not exhibit the distinctive parabolic shape (Fig. 6), neither the turning back behavior revealed by PC3 in the raw spectra.

The hypothesis is also substantiated by the similarities between loadings from the PCA performed on the raw and on the difference spectra (Fig. 7). The loading profile for PC1 in the raw spectra is very similar to that of PC2 in the difference spectra, and the loading pattern of PC2 in the raw spectra is very similar to that of PC1 in the difference spectra. This concurs with preceding findings revealing that the changes caused by temperature are described by PC1 $(96.8 \%)$ in the raw spectra, but by PC2 (10.0\%) in the difference spectra. Similar loading patterns imply that the PCs are describing similar phenomena. Regarding PC3, the loading patterns for the raw and the difference spectra are different, indicating that PC3 does not describes comparable phenomenon in the two circumstances. This agrees with the previous finding stating that PC3 describes the secondary effect of temperature in the raw spectra, but it describes mostly noise in the difference spectra.

The inversion in the rank of the perturbing factors of water (temperature and phospholipid) concurrently with the similarity between the different PC loadings strongly suggest that the main effect of temperature, mirrored mostly by the water molecules in the bulk, is missing after the subtraction of the pure water spectra. Next, the phospholipids become the most important perturbing factor.

\section{Discussion}

\subsection{The main effect of temperature}

The removal of the pure water spectra entailed the elimination of 


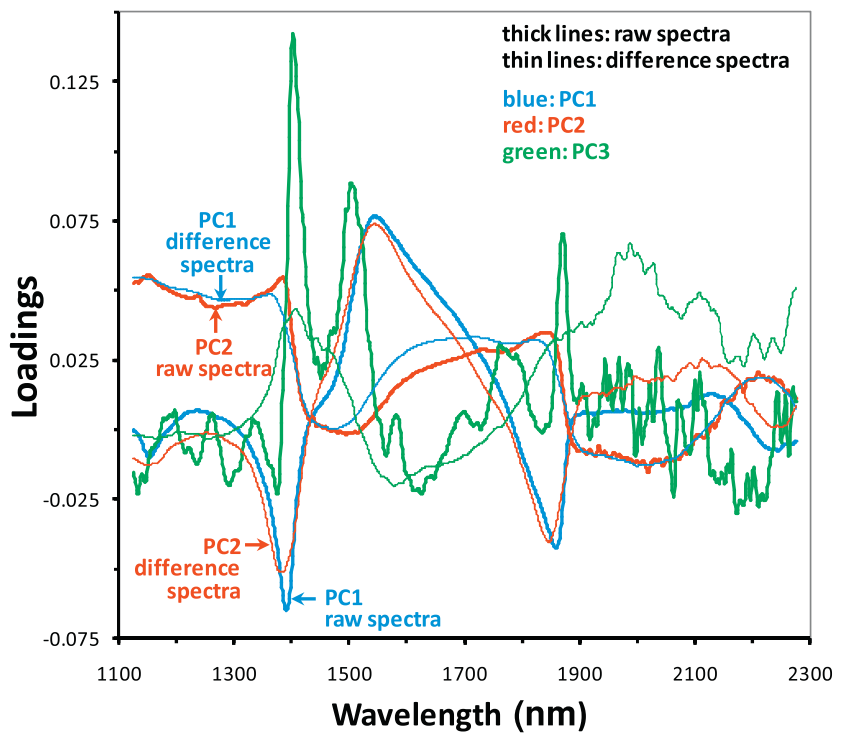

Fig. 7. Comparison of the loadings from PCA performed on the raw (thick series) and difference (thin series) spectra in the $1100-2300 \mathrm{~nm}$ region. PC loadings are identified with the same color in both groups of spectra: PC1, blue; PC2, red; PC3, green. (For interpretation of the references to color in this figure legend, the reader is referred to the web version of this article.)

the major source of spectral variation, i.e. the main effect of temperature. Temperature strongly affects NIR spectrum of water as debilitates the H-bond network and thus it leads to variations in the vibrational energy of the $\mathrm{O}-\mathrm{H}$ bonds. It was reported that changing temperature by $20{ }^{\circ} \mathrm{C}$ had an effect on the H-bond network even greater than adding salts up to $1 \mathrm{~mol}$ concentration [29]. The intensities in the difference spectra (Fig. 5), which mirror the effect of phospholipids, are lower than those of the raw spectra and the signal to noise ratio is also lower. The $1.3 \%$ of variability explained by PC3 in the difference spectra, mainly describing noise (see Section 3.5. The main effect of temperature is missing by removing the pure water), might be representing even less than the $0.1 \%$ of the variability explained by PC3 in the raw spectra, which undoubtedly describes a secondary effect of temperature (Fig. 2B). This rationale may explain why the effect of phospholipids accounts for the $2.9 \%$ of the variability in the raw spectra (described by PC2), whereas such effect account for as much as the $87.6 \%$ of variability in the difference spectra (described by PC1).

The substances inducing a change in the spectrum comparable to an increase in temperature have been termed "structure-breakers", and the substances inducing a change comparable to a decrease of temperature have been termed "structure-makers" [7], a feature early described and named as "order-destroying" and "order-producing" [11]. The effect of the temperature found in this work have also been reported in aqueous solutions of sugars, where the first PC described the effect of temperature and the second one discriminated between different sugars, as glucose, fructose, sucrose and lactose [30].

\subsection{The effect of phospholipids}

The structural traits of a macromolecule for being a "structurebreaker" or a "structure-maker" are not clear. The shift of the $\mathrm{O}-\mathrm{H}$ vibration band to higher frequencies (i.e. higher energy) observed in the presence of DMPA or DMPC implicates the weakening of the $\mathrm{H}$ bonding network and indicates that the phospholipids act as "structurebreakers". More evident for DMPC than for DMPA, the effect augments with phospholipid concentration. The two lipids have identical acyl chains (myristoyl; 14:0) and only differ in their polar head. The presence of a choline $\left[-\mathrm{O}-\mathrm{CH}_{2}-\mathrm{CH}_{2} \mathrm{~N}^{+}\left(\mathrm{CH}_{3}\right)_{3}\right]$ instead of a $[-\mathrm{OH}]$ attached to the phosphate in the polar head of DMPC may explain, at least partially, why DMPC has a disrupting effect on $\mathrm{H}$-bonds stronger than DMPA. The $[-\mathrm{OH}]$ in DMPA may act as a H-bond donor, whereas choline does not. Although highly organized ice-like structures of water with stable aggregates having less free $[-\mathrm{OH}]$ have been found around charged macromolecules [7], the statement might be not so straightforward as the effect of a solute could be opposed depending on its concentrations. Two types of concentration-dependent spectral changes have been described for aqueous solutions of lactose, as the same solute behave as a "structure-maker" at $10-100 \mathrm{mM}$ and as a "structurebreaker" at $0.02-10 \mathrm{mM}$, increasing the amount of free water [30].

The closeness between the phospholipids and pure water series with increasing temperatures indicates that the effect of phospholipids becomes relatively less important with increasing temperatures, and contribute less to the overall disruption of the H-bond network (Fig. 2A). When an increasing proportion of H-bonds is disrupted by increasing temperature, the disrupting effect of phospholipids becomes less significant and it is masked by the dominating "structure-breaker" effect of temperature. For example, almost no additional H-bond disruption is expected for the DMPA liposomes at the highest temperatures in relation to the pure water samples.

\subsection{The secondary effect of temperature}

The straight evolution of the main effect of temperature with PC1 (Fig. 2A) in the raw spectra points to the existence of spectral regions having a monotonous variation. PC3 captures a secondary effect exhibiting a parabolic dependence with temperature and a turning point around $33-38{ }^{\circ} \mathrm{C}$, depending on the solute. It is worth noticing that the turning point is located in the middle of the assayed interval $\left(13-58^{\circ} \mathrm{C}\right)$ just by chance, as it was not previously suspected in the experimental design. A similar parabolic pattern of scores with a turning point at $38^{\circ} \mathrm{C}$ was reported in studies of water aggregates in pure water [24,29]. A comparable increase in the third PC scores at temperatures around $37^{\circ} \mathrm{C}$ was found by Gowen and co-workers [26] in aqueous solutions of inorganic salts $\left(\mathrm{NaCl}, \mathrm{KCl}, \mathrm{MgCl}_{2}\right.$ and $\mathrm{AlCl}_{3}$ ). No unambiguous explanation of this intriguing behavior can be offered here, neither in the cited works. Also interesting is that the secondary effect was also captured in the restricted $1100-1300 \mathrm{~nm}$ interval (Fig. 3B). Further work is needed to elucidate the phenomena behind these findings.

Concurring with the present findings, Segtnan and co-workers [24] also reported a high percentage of variation explained by the principal component that describes the main effect of temperature, PC1 (99.4\%), in relation to PC2 $(0.4 \%)$ which captures the less important, second effect of temperature. As their experimental system was pure water, free of phospholipids and other perturbing components, the second principal component PC2 is focused in the description the second effect of temperature, i.e. the second source of variation. In the actual membranes, the effect of phospholipids is less significant than the main effect of temperature, although greater than the secondary effect of temperature. Then, it is captured by PC2 whereas PC3 is reserved for the second effect of temperature. It is worth noticing that no particular spectral changes associated with the $\mathrm{OH}$ stretching of the water were registered at or near the transition temperatures of phospholipids, i.e. around $50{ }^{\circ} \mathrm{C}$ for DMPA or $24^{\circ} \mathrm{C}$ for DMPC. If the objective is to find the chain-melting phase transitions the analysis has to be focused on the $\mathrm{CH}_{2}$ stretching region, as it was carried out by Kuc and co-workers $[8,9]$. By exploring specifically the $1684-1800 \mathrm{~nm}$ spectral windows, the authors were able to assess the $\mathrm{CH}_{2}$ stretching of the aliphatic chains and thus to capture the phase transition of the phospholipids.

As discussed in Section 3.5. The main effect of temperature is missing by removing the pure water, the loadings pattern for PC3 (Fig. 7) in the difference spectra does not match that in the raw spectra, indicating that PC3 is describing different changes. This supports the assumption that PC3 describes the secondary effect of temperature in the raw spectra, but it does not capture temperature neither phospholipid effect in the difference spectrum, but mainly noise (data not 
shown). The parabolic pattern observed in the PC3/PC1 score plots (Fig. 2B and Fig. 4B) and in PC3/PC2 score plot (Fig. 3B) is missing in the difference spectra.

As mentioned, the two principal components revealing the secondary effect of temperature in the score plots describe temperature effects, i.e. none describe phospholipid effects. This condition is fulfilled in the PC3/PC1 score plot for the $1100-2300 \mathrm{~nm}$ interval (Fig. 2B), in the PC3/PC1 plot for PCA performed on separated groups of samples (Fig. 4B) and in the PC3/PC2 in the 1100-1300 nm interval (Fig. 3B). Leaving the main effect of temperature out by the subtraction of the pure water spectra, PC1 is focused on the description of the effect of phospholipids in both the 1100-2300 (Fig. 6A) and 1100-1300 nm intervals (Fig. 6B), whereas PC2 captures the temperature effects (Fig. 6). Under such circumstances, there is only one PC (PC2) describing temperature effects. This might explain why the characteristic parabolic pattern revealed in the two temperature-depending PCs score plot in the raw spectra is missing and so the effect of temperature is poorly revealed. The need of two PCs describing temperature effects for a clear detection of the secondary effect in the score plot might indicate an interrelation between the two temperature effects. More work is needed to clarify this subject.

\subsection{Populations of water molecules}

The NIR signal emerges from water molecules at the lipid-water interface and from those molecules in the bulk, distant from the phospholipids and perturbed only by the temperature. The current findings point to the presence of two populations of water molecules differentially affected by temperature and phospholipids. The number of molecules in the bulk is clearly larger than that in the interface, and so contributes most to the overall signal. By removing the pure water spectra from the phospholipid-containing membranes the bulk water signal is removed, and the difference spectra inform only about the water molecules at the interface, perturbed by phospholipids. The main effect of temperature on the bulk molecules masks the effect of the phospholipids on the molecules at the interface, a condition that is circumvented by the removal of the pure water spectra.

The missing main effect of temperature in the difference spectra may be explained if considering that all water molecules undergoes the main effect of temperature, i.e. those in the bulk and those at the interface. The difference spectra will depend on the relative size of both populations. The main effect of temperature is detected when a sufficient number of molecules is being measured, i.e. those in the bulk (raw spectra). Once this signal is removed, the relative small number of molecules at the lipid-water interface does not suffice to generate a measurable signal that reveals the main effect of temperature. According to this hypothesis, molecules at the interface undergo the effect of phospholipids more than the effect of temperature. The minimum number of water molecules per lipid $\left(\mathrm{n}_{\mathrm{w}}\right)$ in the gel phase of DMPC required for preventing dramatic physical and structural changes is 12 [31], whereas the number of tightly bound waters per lipid, also namely steric number of waters/lipids ( $\mathrm{nw}^{\prime}$ ), is between 3 and 4.5 $[27,32]$. The current proposal of two water populations is supported by the comparison of the water:lipid molar ratio. It can be easily ascertained that the degree of membrane hydration is quite higher than the aforementioned, and so the amount of water molecules in the bulk. For example, $\mathrm{n}_{\mathrm{w}}$ for the most concentrated lipid suspension $(500 \mu \mathrm{M})$, i.e. that with the lowest water content, is 111,200 . This number of water/ lipid is more than a hundred thousand times greater than the number of waters tightly associated to a phospholipid at the interface (i.e. 3-4.5). Thus, the contribution of the bulk water to the overall signal is the most important. It has been reported [33] that the properties of water beyond the primary hydration layer are basically the same as those of bulk water, and thus there are practically no further hydration layers that can be perturbed by phospholipids except the first one. The water molecules tightly fit into the free spaces of the bilayer in a complex network of H-bonds which may include also the phosphate and carbonyl groups of phospholipids [27].

The characterization of the water species based on the degree of polymerization and the $\mathrm{H}$-bond network as a function of temperature and other "structure-breaker"/"structure-maker" compounds remains unclear. From analysis of the fundamental $\mathrm{O}-\mathrm{H}$ stretching band in the IR region, Binder [27] has reported a simplified two-state model of well-structured, low density "network" water and a less-structured dense "multimer" water, although the author also states a rather continuous change of the water properties with increasing distance from the membrane and with changing temperature. By the simultaneous analysis of fundamental stretching and bending vibrations of the $\mathrm{O}-\mathrm{H}$ by using both NIR and MIR spectroscopy, Czarnik-Matusewicz and coworkers [34] also proposed a two-component structure of water. Not distant from these proposals, a somewhat distorted two-state structural model for water was suggested by Segtnan and co-workers [24]. The authors found that the wavelengths 1412 and $1491 \mathrm{~nm}$ account for > $99 \%$ of the spectral variation and represent two major water species with weaker and stronger hydrogen bonds, respectively. They also indicate the presence of a third species absorbing at $1438 \mathrm{~nm}$, whose concentration was relatively constant as a function of temperature. Alternatively, from the analysis of 1441-1470 nm band in the NIR region, Luck [35] claim the existence of water molecules having H-bonds with one or two other water molecules, i.e. implying the existence of water dimers and trimers. Also from NIR spectroscopy data, Maeda and co-workers [23] reported five spectral components, and each one was ascribed to the water species with no, one, two, three and four hydrogen bonds.

Much work remains to be done to ascertain an unambiguous picture of the water in membranes and the perturbations caused by temperature and phospholipids. The intriguing turning point of the secondary effect of temperature around $33-38{ }^{\circ} \mathrm{C}$ and the need of two temperature-associated PCs for a clear detection the secondary effect are also questions to be answered.

\section{Financial and competing interest disclosure}

This research did not receive any specific grant from funding agencies in the public, commercial, or not-for-profit sectors. No writing assistance was utilized.

\section{Transparency document}

The Transparency document associated with this article can be found, in online version.

\section{Acknowledgment}

I thank Silvia Barboza for providing the near infrared analyzer and Javier Vitale for the help in their use.

\section{Appendix A. Supplementary data}

Supplementary data to this article can be found online at https:// doi.org/10.1016/j.bbamem.2017.12.007.

\section{References}

[1] G.L. Jendrasiak, J.H. Hasty, The hydration of phospholipids, Biochim. Biophys. Acta 337 (1974) 79-91.

[2] V. Luzzati, T. Gulik-Krzywicki, F. Rivas, F. Reiss-Husson, R.P. Rand, X-ray study of model systems: structure of the lipid-water phases in correlation with the chemical composition of the lipids, J. Gen. Physiol. 51 (1968) 37-43.

[3] E.G. Finer, A. Darke, Phospholipid hydration studied by deuteron magnetic resonace spectroscopy, Chem. Phys. Lipids 12 (1974) 1-16.

[4] V.J. Frost, K. Molt, Analysis of aqueous solutions by near-infrared spectrometry (NIRS) III. Binary mixtures of inorganic salts in water, J. Mol. Struct. 410 (1997) 573-579. 
[5] K. Buijs, G. Choppin, Near-infrared studies of the structure of water. I. Pure water, J. Chem. Phys. 39 (1963) 2035-2041.

[6] M. Golic, K. Walsh, P. Lawson, Short-wavelength near-infrared spectra of sucrose, glucose, and fructose with respect to sugar concentration and temperature, Appl. Spectrosc. 57 (2003) 139-145.

[7] R. Giangiacomo, Study of water-sugar interactions at increasing sugar concentration by NIR spectroscopy, Food Chem. 96 (2006) 371-379.

[8] M. Kuć, K. Cieślik-Boczula, M. Rospenk, Influence of inhalation anesthetics on the chain-melting phase transition of DPPC liposomes. Near-infrared spectroscopy studies supported by PCA analysis, Vib. Spectrosc. 85 (2016) 55-61.

[9] M. Kuć, K. Cieślik-Boczula, M. Rospenk, Anesthetic-dependent changes in the chainmelting phase transition of DPPG liposomes studied using near-infrared spectroscopy supported by PCA, Spectrochim. Acta A Mol. Biomol. Spectrosc. 186 (2017) $37-43$.

[10] J. Collins, Change in the infra-red absorption spectrum of water with temperature, Phys. Rev. 26 (1925) 771.

[11] G. Choppin, K. Buijs, Near-infrared studies of the structure of water. II. Ionic solutions, J. Chem. Phys. 39 (1963) 2042-2050.

[12] R. Tsenkova, Aquaphotomics: dynamic spectroscopy of aqueous and biological systems describes peculiarities of water, J. Near Infrared Spectrosc. 17 (2009) 303-314.

[13] R.N. Tsenkova, I.K. Iordanova, K. Toyoda, D.R. Brown, Prion protein fate governed by metal binding, Biochem. Biophys. Res. Commun. 325 (2004) 1005-1012.

[14] F.J. Rambla, S. Garrigues, M. de la Guardia, PLS-NIR determination of total sugar, glucose, fructose and sucrose in aqueous solutions of fruit juices, Anal. Chim. Acta 344 (1997) 41-53.

[15] G. Bazar, R. Romvari, A. Szabo, T. Somogyi, V. Eles, R. Tsenkova, NIR detection of honey adulteration reveals differences in water spectral pattern, Food Chem. 194 (2016) 873-880.

[16] G. Takemura, G. Bazar, K. Ikuta, E. Yamaguchi, S. Ishikawa, A. Furukawa, Y. Kubota, Z. Kovacs, R. Tsenkova, Aquagrams of raw milk for oestrus detection in dairy cows, Reprod. Domest. Anim. Zuchthygiene 50 (2015) 522-525.

[17] N. Goto, G. Bazar, Z. Kovacs, M. Kunisada, H. Morita, S. Kizaki, H. Sugiyama, R. Tsenkova, C. Nishigori, Detection of UV-induced cyclobutane pyrimidine dimers by near-infrared spectroscopy and aquaphotomics, Sci. Rep. 5 (2015) 11808.

[18] B. Jinendra, K. Tamaki, S. Kuroki, M. Vassileva, S. Yoshida, R. Tsenkova, Near infrared spectroscopy and aquaphotomics: novel approach for rapid in vivo diagnosis of virus infected soybean, Biochem. Biophys. Res. Commun. 397 (2010) 685-690.

[19] R. Tsenkova, S. Atanassova, S. Kawano, K. Toyoda, Somatic cell count determination in cow's milk by near-infrared spectroscopy: a new diagnostic tool, J. Anim. Sci. 79 (2001) 2550-2557.

[20] K.R. Beeve, R.J. Pell, M.B. Seasholtz, Chemometrics: A Practical Guide, John Wiley
\& Sons, New York, 1998.

[21] R.G. Brereton, Chemometrics: Data Analysis for the Laboratory and Chemical Plant, John Wiley \& Sons, England, 2003.

[22] The Unscrambler, Camo Process As, Oslo, Norway, http://www.camo.no, (2007).

[23] H. Maeda, Y. Ozaki, M. Tanaka, N. Hayashi, T. Kojima, Near infrared spectroscopy and chemometrics studies of temperature-dependent spectral variations of water: relationship between spectral changes and hydrogen bonds, J. Near Infrared Spectrosc. 3 (1995) 191-201.

[24] V.H. Segtnan, S. Sasic, T. Isaksson, Y. Ozaki, Studies on the structure of water using two-dimensional near-infrared correlation spectroscopy and principal component analysis, Anal. Chem. 73 (2001) 3153-3161.

[25] W.C. McCabe, S. Subramanian, H.F. Fisher, Near-infrared spectroscopic investigation of the effect of temperature on the structure of water, J. Phys. Chem. 74 (1970) 4360-4369.

[26] A.A. Gowen, J.M. Amigo, R. Tsenkova, Characterisation of hydrogen bond perturbations in aqueous systems using aquaphotomics and multivariate curve resolutionalternating least squares, Anal. Chim. Acta 759 (2013) 8-20.

[27] H. Binder, Water near lipid membranes as seen by infrared spectroscopy, Eur. Biophys. J. EBJ 36 (2007) 265-279.

[28] J.J. Wenz, Molecular properties of steroids involved in their effects on the biophysical state of membranes, Biochim. Biophys. Acta 1848 (2015) 2448-2459.

[29] R. Tsenkova, Z. Kovacs, Y. Kubota, Aquaphotomics: near infrared spectroscopy and water states in biological systems, Subcell. Biochem. 71 (2015) 189-211.

[30] G. Bazar, Z. Kovacs, M. Tanaka, A. Furukawa, A. Nagai, M. Osawa, Y. Itakura, H. Sugiyama, R. Tsenkova, Water revealed as molecular mirror when measuring low concentrations of sugar with near infrared light, Anal. Chim. Acta 896 (2015) $52-62$.

[31] S. Tristram-Nagle, Y. Liu, J. Legleiter, J.F. Nagle, Structure of gel phase DMPC determined by X-ray diffraction, Biophys. J. 83 (2002) 3324-3335.

[32] S. Tristram-Nagle, Use of X-ray and neutron scattering methods with volume measurements to determine lipid bilayer structure and number of water molecules/ lipid, in: A.D. (Ed.), Membrane Hydration: The Role of Water in the Structure and Function of Biological Membranes, Springer, Switzerland, 2015.

[33] J. Israelachvili, H. Wennerstrom, Role of hydration and water structure in biological and colloidal interactions, Nature 379 (1996) 219-225.

[34] B. Czarnik-Matusewicz, S. Pilorz, J.P. Hawranek, Temperature-dependent water structural transitions examined by near-IR and mid-IR spectra analyzed by multivariate curve resolution and two-dimensional correlation spectroscopy, Anal. Chim. Acta 544 (2005) 15-25.

[35] W.A.P. Luck, The importance of cooperativity for the properties of liquid water, J. Mol. Struct. 448 (1998) 131-142. 